\title{
An Ultraviolet Hybrid Plasmonic Waveguide for Nanolaser Applications
}

\author{
Zhiquan Li' ${ }^{1}$, Yajuan Wang1, Jiahuan $\mathrm{He}^{1}$, Dandan Feng ${ }^{1}$, Erdan $\mathrm{Gu}^{1}$, Wenchao $\mathrm{Li}^{2}$ \\ ${ }^{1}$ Institute of Electrical Engineering, Yanshan University, Qinhuangdao, China \\ ${ }^{2}$ School of Control Engineering, Northeastern University at Qinhuangdao, Qinhuangdao, China \\ Email: Izq54@ysu.edu.cn
}

Received 20 April 2016; accepted 19 August 2016; published 25 August 2016

\begin{abstract}
In this paper, a novel hybrid plasmonic waveguide with a metal ridge and an MgF2 dielectric layer is demonstrated at ultraviolet band. We investigate the propagation distance, the scaling factor and the figure of merit by using the finite element method. The structure enables low scaling factor and long propagation distance. Compared to the previous structure with a metal plate, this waveguide has better performance. And the structure can be used as a nanolaser and has broad application prospects in optoelectronic integrated circuits, biological detection and so on.
\end{abstract}

\section{Keywords}

Ultraviolet, Plasmonic, Waveguide

\section{Introduction}

In recent years, surface plasmons are introduced to break diffraction limit of waveguide, whose size must be larger than the half wavelength of the optical field in all three dimensions [1]. In this way, plasmonic waveguide can reach subwavelength optical confinement [2] by using the surface plasmon polaritons, which are Transverse Magnetic (TM) polarized surface wave propagating along metal-dielectric interfaces [3]. Therefore, various types of plasmonic waveguide have been presented, such as metal-insulator-metal (MIM) [4], long-range SPP (LSPP) [5], metallic nanowire [6] waveguides and hybrid plasmonic waveguide [7]. However, the previous reports on plasmonic waveguide almost achieved at visible and infrared region because of the lower metal absorption [8]. From the developing trend of the lasing, the main direction goes forward to short wavelength [9], such as ultraviolet band. It benefits to increase storage density of optical information and bandwidth of optical communication [10] [11]. In addition, the ultraviolet resonance Raman spectroscopy is an important means to detect biological molecules [12]. In this paper, we propose a novel hybrid plasmonic waveguide at ultraviolet band. The structure shows tight field confinement and long propagation distance, and reaches deep sub-wavelengthscale. The design has promising potential for application in nanolaser, plasmonic systems and biological detection.

\section{Structural Design and Simulation}

The geometry of the proposed waveguide is shown in Figure 1(a). The structure consists of a Al metal ridge, a

How to cite this paper: Li, Z.Q., Wang, Y.J., He, J.H., Feng, D.D., Gu, E.D. and Li, W.C. (2016) An Ultraviolet Hybrid Plasmonic Waveguide for Nanolaser Applications. Optics and Photonics Journal, 6, 19-23.

http://dx.doi.org/10.4236/opj.2016.68B004 

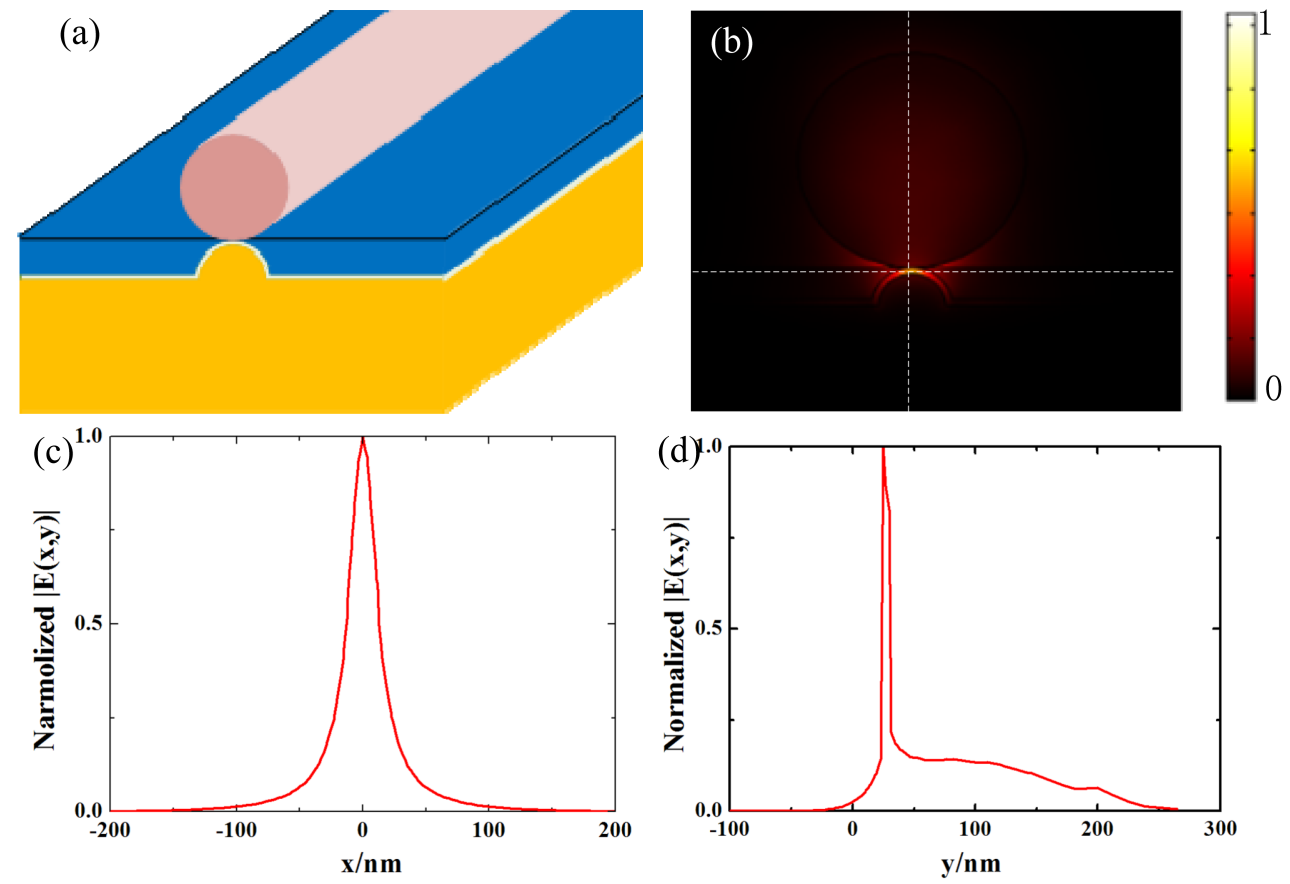

Figure 1. (a) Geometry of the proposed waveguide; (b) Normalized electric field distribution of the fundamental hybrid plasmonic mode of the proposed structure; (c) and (d) Normalized electric field distribution along the horizontal and vertical dashed lines in (b) $(r=80 \mathrm{~nm})$.

low-index $\mathrm{MgF}_{2}$ dielectric layer, a $\mathrm{SiO}_{2}$ layer and a high-index $\mathrm{GaN}$ nanowire. The width of the metal layer is $300 \mathrm{~nm}$, and its height is $100 \mathrm{~nm}$. The length of the GaN nanowire $L$ is $30 \mu \mathrm{m}$, its radius is $r$. The radius of the metal ridge is fixed at $35 \mathrm{~nm}$. The thickness of the $\mathrm{MgF}_{2}$ dielectric layer is $5 \mathrm{~nm}$. At the working wavelength of $370 \mathrm{~nm}$, the refractive indices of $\mathrm{Al}, \mathrm{MgF}_{2}, \mathrm{SiO}_{2}$ and $\mathrm{GaN}$ are $0.38829+4.3466 \mathrm{i}, 1.3856,1.46$ and 2.65, respectively [11] [13].

Figure 1(b) shows the electric field distribution of the fundamental hybrid plasmonic mode of the proposed structure, where the geometric parameters are chosen as $r_{1}=35 \mathrm{~nm}, r_{3}=80 \mathrm{~nm}, t=5 \mathrm{~nm}$. The field enhancements in the horizontal and vertical directions are shown in Figure 1(c) and Figure 1(d), respectively. By introducing a metal ridge and the filmy $\mathrm{MgF}_{2}$ dielectric layer into the design, the electric field energy is concentrated in a tiny area.

To quantify the mode properties, we introduce the indices of the mode scaling factor ( $S F$ ) and the propagation distance $(D)$ [14]. The $S F$ is calculated as the ratio of the effective mode area to the diffraction-limited mode area. It represents the confinement ability for the mode field which is expressed [14] as

$$
S F=A_{\text {eff }} / A_{0}
$$

Here, the effective mode area $A_{\text {eff }}$ and the diffraction-limited mode area $A_{0}$ are expressed by using the following formulas [15]:

$$
\begin{gathered}
A_{e f f}=\left(\iint|\mathrm{E}|^{2} d x d y\right)^{2} /\left(\iint|\mathrm{E}|^{4} d x d y\right) \\
A_{0}=\lambda^{2} / 4
\end{gathered}
$$

In the above expressions, $E$ is the electric field intensity of the hybrid mode and $\lambda$ is the working wavelength. The propagation distance $(D)$ is defined as [11]

$$
D=\frac{1}{2 \operatorname{Im}(\beta)}
$$

where $\beta$ is the mode propagation constant. 
The Figure 2 shows the effect of the nanowire radius $(r)$ on the performances of the structure. Obviously, the mode scaling factor firstly decreases before increaseing while the propagation distance declines with the nanowire radius increasing. When the nanowire radius approaches the metal rib radius, the mode scaling factor achieves the minimum value 0.0194 . In the case, the effective mode area is $0.00485 \lambda^{2}$. The larger nanowire radius leads to larger propagation distance, but larger mode scaling factor. So it is meaningful to define the figure of merit ( FOM ), which is given by [14]

$$
F O M=\frac{D}{S F}
$$

The lager FOM indicates better performance of a waveguide. The Figure 3 shows the FOM with the nanowire radius increasing firstly increases before decreasing. The maximum value $28.56 \mu \mathrm{m}$ is obtained at $r=80 \mathrm{~nm}$. In addition, it indicates the FOM of the present structure is larger than that of previous structure with a metal plate [12], owing to the metal ridge and the filmy $\mathrm{MgF}_{2}$ dielectric layer.

The pump threshold is the minimal value the gain reaches when achieving lasing action. It is related to the nanowire length $L$ and the end facet reflectivity $R$. The $R$ [16] is expressed by the following equation:

$$
R=\left(n_{\text {eff }}-1\right) /\left(n_{\text {eff }}+1\right)
$$

The lasing threshold is calculate [16] by

$$
G \text { th }=\left(k_{0} \partial_{\text {eff }}+\ln (1 / R) / L\right) / \Gamma^{*}\left(n_{\text {eff }} / n_{\text {wire }}\right)
$$

where $k_{0}=2 \pi / \lambda, n_{\text {wire }}$ is the refractive index of the gain nanowire, and $n_{\text {eff }} / n_{\text {wire }}$ is the enhancement part of the modal effective index. Figure 4 shows that the pump threshold of the proposed waveguide increases with enlarging $r$. Its minimum is $0.328 \times 10^{3} \mathrm{~cm}^{-1}$.

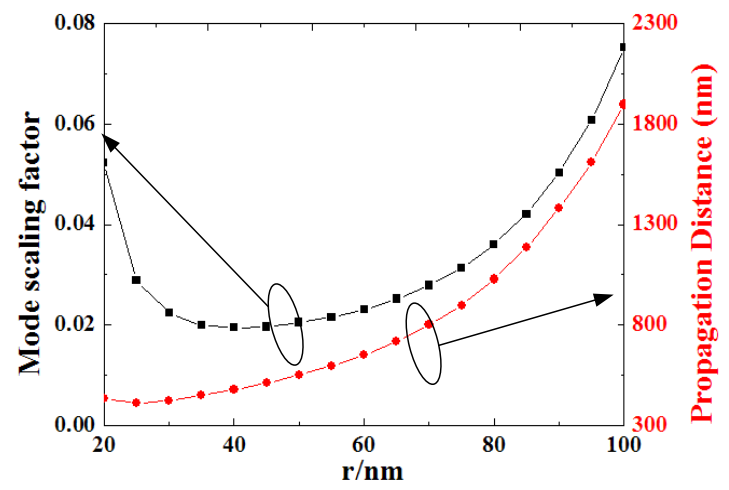

Figure 2. The $S F$ and $D$ of the fundamental hybrid plasmonic mode with different $r$.

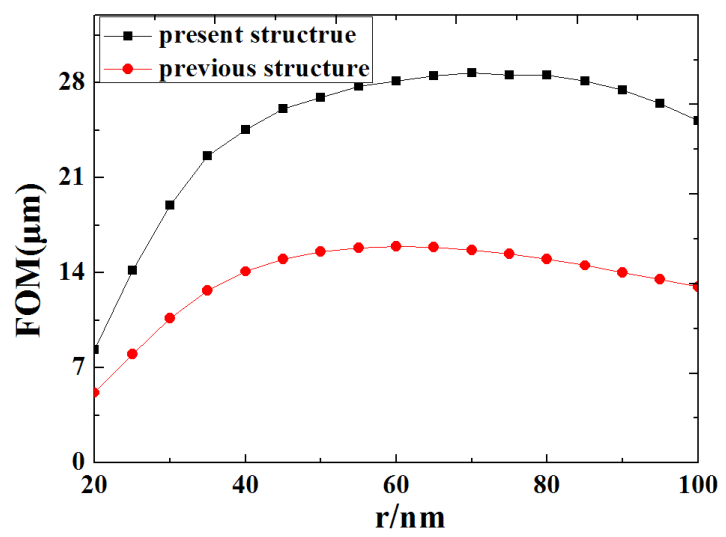

Figure 3. The FOM of the fundamental hybrid plasmonic mode of present structure and previous structure with different $r$. 


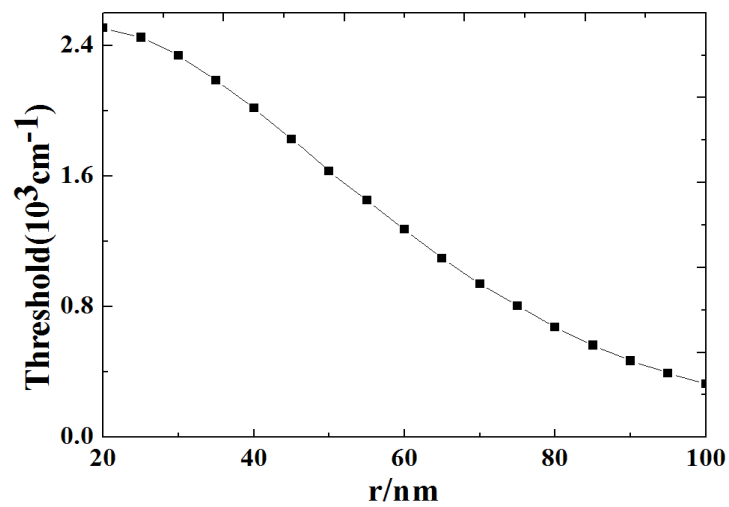

Figure 4. The pump threshold of the fundamental hybrid plasmonic mode of present structure with different $r$.

\section{Conclusion}

We present a new type of ultraviolet waveguide based on surface plasmons which attains deep-subwavelength scale and has long propagation distance. By using the COMSOL Multiplicity software, we investigate the light field distribution, and analyze the effect of the radius of the gain medium nanowire on the properties and the lasing threshold. The results show that the larger nanowire radius causes the better performance of the proposed waveguide. So we can select the optimal radius of the nanowire as $80 \mathrm{~nm}$. In this case, the $S F$, the $D$ and the threshold are $0.0359,1027.3 \mathrm{~nm}$ and $0.67 \times 10^{3} \mathrm{~cm}^{-1}$, respectively. Compared to the previous ultraviolet waveguide with a metal plate, the performance of the present structure with a metal redge is improved significantly with the same geometric parameters. The designed structure offers a new idea for the high-density photonic integrated devices, such as the deep-subwavelength-scale ultraviolet nanolaser.

\section{Acknowledgements}

We acknowledge support from the Hundred-Talent Program of Hebei Province and the Natural Science Foundation of Hebei Province in China.

\section{References}

[1] Zhu, L. (2010) Modal Properties of Hybrid Plasmonic Waveguides for Nanolaser Applications. IEEE Photonics Technology Letters, 22, 535-537. http://dx.doi.org/10.1109/LPT.2010.2041923 http://ieeexplore.ieee.org/xpls/abs_all.jsp?arnumber=5418941\&tag=1

[2] Gramotnev, D.K., and Bozhevolnyi, S.I. (2010) Plasmonics beyond the Diffraction Limit. Nature Photonics, 4, 83-91. http://dx.doi.org/10.1038/nphoton.2009.282 http://www.nature.com/nphoton/journal/v4/n2/abs/nphoton.2009.282.html

[3] Barnes, W.L., Dereux, A. and Ebbesen, T.W. (2003) Surface Plasmon Subwavelength Optics. Nature, 424, 824-830. http://dx.doi.org/10.1038/nature01937 http://www.nature.com/nature/journal/v424/n6950/abs/nature01937.html

[4] Hill, M.T., Marell, M., Leong, E.S., Smalbrugge, B., Zhu, Y.C., Sun, M.H., Nötzel, R., et al. (2009) Lasing in MetalInsulator-Metal Sub-Wavelength Plasmonic Waveguides. Optics Express, 17, 11107-11112. http://dx.doi.org/10.1364/OE.17.011107 https://www.osapublishing.org/oe/abstract.cfm?uri=oe-17-13-11107

[5] Holmgaard, T., Gosciniak, J. and Bozhevolnyi, S.I. (2010) Longrange Dielectric-Loaded Surface Plasmon-Polariton Waveguides. Optics Express, 18, 23009-23015. http://dx.doi.org/10.1364/OE.18.023009 https://www.osapublishing.org/oe/abstract.cfm?uri=oe-18-22-23009

[6] Zou, C.I., Sun, F.W., Xiao, Y.F., Dong, C.H., Chen, X.D., Cui, J.M., Gong, Q., Han, Z.F. and Guo, G.C. (2010) Plasmon Modes of Silver Nanowire On A Silica Substrate. Apply Physics Letter, 97, 18310. http://scitation.aip.org/content/aip/journal/apl/97/18/10.1063/1.3509415

[7] Oulton, R.F., Sorger, V.J., Genov, D.A., Pile, D.F.P. and Zhang, X. (2008) A Hhybrid Plasmonic Waveguide for Subwavelength Confinement and Long-Range Propagation. Nature Photonics, 2, 496-500. http://dx.doi.org/10.1038/nphoton.2008.131 http://www.nature.com/nphoton/journal/v2/n8/abs/nphoton.2008.131.html

[8] Chen, Y., Tong, C., Qin, L., Wang, L.J. and Zhang, J.L. (2012) Progress in Surface Plasmon Polariton Nano-Laser 
Technologies and Applications. Chinese Optics, 5, 453-463. http://dx.doi.org/10.3788/co.20120505.0453 http://en.cnki.com.cn/Article_en/CJFDTOTAL-ZGGA201205004.htm

[9] Wu., J.-L. (2005) Study of ZnO Nanoscale Laser with Near Ultraviolet Wavelength. Vacuum Electronics, 6, 001. http://www.cnki.com.cn/Article/CJFDTotal-ZKDJ200506001.htm

[10] Huang, M.H., Mao, S., Feick, H., Yan, H., Wu, Y., Kind, H. and Yang, P. (2001) Room-Temperature Ultraviolet Nanowire Nanolasers. Science, 292, 1897-1899. http://dx.doi.org/10.1126/science.1060367 http://science.sciencemag.org/content/292/5523/1897

[11] Zhang, Q., Li, G., Liu, X., Qian, F., Li, Y., Sum, T.C. and Xiong, Q. (2014) A Room Temperature Low-Threshold Ultraviolet Plasmonic Nanolaser. Nature Communications, 5. http://dx.doi.org/10.1038/ncomms5953 http://www.nature.com/ncomms/2014/140923/ncomms5953/full/ncomms5953.html

[12] Hu, M.Z., Zhou, S.Y., Han, Q., Sun, H., Zhou, L.P., Zeng, C.M., Wu, Z.F. and Wu, X.M. (2014) Ultrviolet Surface Pplasmon Propagation for ZnO Semiconductor-Insulator-Metal Waveguides. Acta Phys. Sin, 63, 029501. http://wulixb.iphy.ac.cn/CN/abstract/abstract56954.shtml

[13] Sharma, A.K. and Gupta, B.D. (2007) On the Performance of Different Bimetallic Combinations in Surface Plasmon Resonance Based Fiber Optic Sensors. Journal of Applied Physics, 101, 093111. http://dx.doi.org/10.1063/1.2721779 http://scitation.aip.org/content/aip/journal/jap/101/9/10.1063/1.2721779

[14] Mu, J., Chen, L., Li, X., Huang, W.P., Kimerling, L.C. and Michel, J. (2013) Hybrid Nano Ridge Plasmonic Polaritons Waveguides. Applied Physics Letters, 103, 131107. http://dx.doi.org/10.1063/1.4823546 http://scitation.aip.org/content/aip/journal/apl/103/13/10.1063/1.4823546

[15] Bian, Y., Zheng, Z., Liu, Y., Zhu, J. and Zhou, T. (2011) Coplanar Plasmonic Nanolasers Based on Edge-coupled Hybrid Plasmonic Waveguides. IEEE Photonics Technology Letters, 23, 884-886. http://ieeexplore.ieee.org/xpls/abs_all.jsp?arnumber=5753919\&tag=1 http://dx.doi.org/10.1109/LPT.2011.2141981

[16] Liu, J.-T., Xu, B.-Z., Zhang, J., Cai, L.-K. and Song, G.-F. (2012) Gain-Assisted Indented Plasmonic Waveguide for Low-Threshold Nanolaser Applications. Chinese Physics B, 21, 107303.

http://dx.doi.org/10.1088/1674-1056/21/10/107303

http://iopscience.iop.org/article/10.1088/1674-1056/21/10/107303/meta

\section{Submit or recommend next manuscript to SCIRP and we will provide best service for you:}

Accepting pre-submission inquiries through Email, Facebook, LinkedIn, Twitter, etc.

A wide selection of journals (inclusive of 9 subjects, more than 200 journals)

Providing 24-hour high-quality service

User-friendly online submission system

Fair and swift peer-review system

Efficient typesetting and proofreading procedure

Display of the result of downloads and visits, as well as the number of cited articles

Maximum dissemination of your research work

Submit your manuscript at: http://papersubmission.scirp.org/ 